\title{
CERTAIN CONTINUED FRACTION REPRESENTATIONS FOR FUNCTIONS ASSOCIATED WITH MOCK THETA FUNCTIONS OF ORDER THREE
}

\author{
Anand Kumar Srivastava
}

\section{Introduction}

The object of this paper is to place on record certain continued fraction representations for partial mock theta functions of order three. We know the continued fraction representations for $a_{2} \Phi_{1}[a, b ; c ; z]$ when $z$ and $a, b, c$ take a particular form. It is not known whether $a_{2} \Phi_{1}[a, b ; c ; z]_{m}$ may also give continued fraction representations.

\section{Notation}

The usual hypergeometric notation will be followed. Thus for $\left|q^{k}\right|<1$, let

$$
\begin{aligned}
& \left(a ; q^{k}\right)_{n}=(1-a)\left(1-a q^{k}\right)\left(1-a q^{2 k}\right) \cdots\left(1-a q^{(n-1) k}\right), \quad n>0, k>0, \\
& \left(a ; q^{k}\right)_{0}=1 .
\end{aligned}
$$

Also let $\left(a_{1}, a_{2}, \ldots, a_{r} ; q^{k}\right)_{n}=\left(a_{1} ; q^{k}\right)_{n}\left(a_{2} ; q^{k}\right)_{n} \cdots\left(a_{r} ; q^{k}\right)_{n}$ and $\left(a ; q^{k}\right)_{\infty}=$ $\prod_{n=0}^{\infty}\left(1-a q^{k n}\right)$.

A basic hypergeometric series ${ }_{r+1} \Phi_{r}$ is defined as

$$
\begin{aligned}
{ }_{r+1} \Phi_{r}^{\left(q^{k}\right)}\left[\begin{array}{c}
\alpha_{1}, \alpha_{2}, \ldots, \alpha_{r+1} ; z \\
\beta_{1}, \beta_{2}, \ldots, \beta_{r}
\end{array}\right] & ={ }_{r+1} \Phi_{r}^{\left(q^{k}\right)}\left[\alpha_{1}, \ldots, \alpha_{r+1} ; \beta_{1}, \ldots, \beta_{r} ; z\right] \\
& =\sum_{n=0}^{\infty} \frac{\left(\alpha_{1}, \alpha_{2}, \ldots, \alpha_{r+1} ; q^{k}\right)_{n} z^{n}}{\left(q^{k}, \beta_{1}, \beta_{2}, \ldots, \beta_{r} ; q^{k}\right)_{n}} .
\end{aligned}
$$

The series (2.1) converges for $|z|<1,|q|<1$.

Further, let

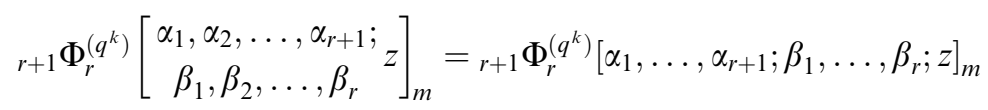

denote the $m^{\text {th }}$ partial sum $\sum_{n=0}^{m}\left(\left(\alpha_{1}, \alpha_{2}, \ldots, \alpha_{r+1} ; q^{k}\right)_{n} z^{n} /\left(q^{k}, \beta_{1}, \beta_{2}, \ldots, \beta_{r}\right.\right.$; $\left.q^{k}\right)_{n}$ ) of the basic hypergeometric series.

Received January 29, 2002. 
Whenever $k=1$, it shall be omitted from all the above symbols including the $\Phi$-symbol.

\section{Definitions of partial mock theta functions of order three, six and Jacobi's theta functions}

N. J. Fine [4] represented Ramanujan's [7, p. 129-130] third order mock theta functions as particular cases of a ${ }_{2} \Phi_{1}[q, \alpha ; \beta ; z]$ as follows:

(i) $\quad f(q)=\frac{2 q}{(1+q)}+\frac{1}{(1+q)}{ }_{2} \Phi_{1}\left[q, o ;-q ;-q^{2}\right]$,

(ii) $\phi(q)=\frac{(2+q-i q)}{(1-i q)}-\frac{1}{(1-i q)}{ }_{2} \Phi_{1}\left[q, o ;-i q ; i q^{2}\right]$,

(iii) $\psi(q)=\frac{q}{(1-q)}+\frac{q^{5}}{(1-q)}{ }_{2} \Phi_{1}^{\left(q^{2}\right)}\left[q^{2},-q^{2} ; o ; q^{3}\right]$,

(iv) $\chi(q)=\frac{\left(1+q-\omega^{2}+\omega^{2} q\right)}{\left(1+\omega^{2} q\right)}+\frac{\omega^{2}}{\left(1+\omega^{2} q\right)}{ }_{2} \Phi_{1}^{\left(q^{2}\right)}\left[q, o ;-\omega q ;-\omega^{2} q^{2}\right]$, where $\omega=e^{2 \pi i / 3}$.

(v) $\omega(q)=\frac{1}{(1-q)}+\frac{q}{(1-q)^{2}}{ }_{2} \Phi_{1}^{\left(q^{2}\right)}\left[q^{2}, o ; q^{3} ; q^{3}\right]$,

(vi) $v(q)=\frac{1}{(1+q)}+\frac{q^{2}}{(1+q)}{ }_{2} \Phi_{1}^{\left(q^{2}\right)}\left[q^{2}, q ; o ;-q^{3}\right]$,

(vii) $\rho(q)=(1-\omega q)+\omega q_{2} \Phi_{1}^{\left(q^{2}\right)}\left[q^{2}, o ; \omega q^{3} ; q^{3} \omega^{-1}\right]$, where $\omega=e^{2 \pi i / 3}$.

Andrews and Hickerson [3] called the following four mock theta functions as of order 'six' without ascribing any reason. R. P. Agarwal and Anju Gupta [5] represented these four mock theta functions in terms of a particular ${ }_{2} \Phi_{1}[q, \alpha ; \beta ; z]$ series in the following form:

(i)

$$
\begin{aligned}
\phi_{L}(q)= & \frac{2(1-\omega)}{3}+\frac{2 \omega(1-\omega)}{3\left(1-\omega^{2} q\right)}+\frac{2 \omega^{2}}{3\left(1-\omega^{2} q\right)}{ }_{2} \Phi_{1}\left[q, o ; \omega q ; \omega^{2} q^{2}\right] \\
& +\frac{1}{3} \frac{j\left(q ; q^{2}\right)^{2}}{j\left(-q ; q^{3}\right)}
\end{aligned}
$$

(ii) $\sigma_{L}(q)=\frac{1}{2}\left(-q ; q^{2}\right)_{\infty}^{2} j\left(-q^{3} ; q^{6}\right)-\frac{1}{6} \frac{j\left(q^{2} ; q^{4}\right)^{2}}{j\left(-q^{2} ; q^{6}\right)}-\frac{(1-\omega)}{3}-\frac{\omega(1-\omega)}{3\left(1-\omega^{2} q^{2}\right)}$

$$
-\frac{\omega^{2}}{3\left(1-\omega^{2} q^{2}\right)}{ }_{2} \Phi_{1}^{\left(q^{2}\right)}\left[q^{2}, o ; \omega q^{2} ; \omega^{2} q^{4}\right]
$$




$$
\begin{aligned}
& \text { (iii) } \mu_{L}(q)=\frac{2(1-\omega)}{3}+\frac{2 \omega(1-\omega)}{3\left(1-\omega^{2} q^{2}\right)}+\frac{2 \omega^{2}}{3\left(1-\omega^{2} q^{2}\right)}{ }_{2} \Phi_{1}^{\left(q^{2}\right)}\left[q^{2}, o ; \omega q^{2} ; \omega^{2} q^{4}\right] \\
& +\frac{1}{3} \frac{j\left(q^{2} ; q^{4}\right)^{2}}{j\left(-q^{2} ; q^{6}\right)}-\frac{1}{2}\left(q ; q^{2}\right)_{\infty}^{2} j\left(q^{3} ; q^{6}\right), \\
& \text { (iv) } \gamma_{L}(q)=-\frac{2 \omega^{2}}{(1+\omega)}+\frac{\omega^{2}}{\left(1-\omega^{2}\right)}{ }_{2} \Phi_{1}\left[q, o ; \omega q ; \omega^{2} q^{2}\right] \text {. }
\end{aligned}
$$

where $j\left(x, q^{k}\right):=\left(x, q^{k}\right)_{\infty}\left(q^{k} / x, q^{k}\right)_{\infty}\left(q^{k}, q^{k}\right)_{\infty}$ and suffix $L$ denotes that these mock theta functions were found by Andrews in the 'Lost' Notebook. Agarwal [1] in view of his definition of the order of a mock theta function designated the above four functions (3.2) as belonging to order three.

The classical three out of four Jacobi's theta functions [6, p. 324-325] are defined as:

(i) $\quad \theta_{2}(0)=2 q^{1 / 4} \sum_{n=0}^{\infty} q^{n(n+1)}$,

(ii) $\theta_{3}(0)=1+2 \sum_{n=0}^{\infty} q^{n^{2}}$

(iii) $\theta_{4}(0)=1+2 \sum_{n=0}^{\infty}(-1)^{n} q^{n^{2}}$.

These three theta functions are limiting cases of ${ }_{2} \Phi_{1}[q, \alpha ; \beta ; z]$.

The partial sums of the series (3.1(i-vii)), (3.2(i-iv)) and (3.3(i-iii)) by taking ${ }_{2} \Phi_{1}[q, \alpha ; \beta ; z]_{m}=\sum_{n=0}^{m}\left((\alpha)_{n} z^{n} /(\beta)_{n}\right)$ called the partial mock theta functions of order three, six and partial theta functions be denoted by $f_{m}(q), \phi_{m}(q), \psi_{m}(q)$, $\chi_{m}(q), \omega_{m}(q), v_{m}(q), \rho_{m}(q), \phi_{L m}(q), \sigma_{L m}(q), \mu_{L m}(q), \gamma_{L m}(q), \theta_{2, m}(o), \theta_{3, m}(o)$, $\theta_{4, m}(o)$ respectively.

\section{An identity connecting series of the type $\sum_{n=0}^{m}\left((\alpha)_{n} z^{n} /(\beta)_{n}\right)$}

It is not difficult to prove, by series manupulation, the identity

$$
\begin{gathered}
(1-t) \sum_{n=0}^{m} \frac{(a q)_{n} t^{n}}{(b q)_{n}}-(b-a t q) \sum_{n=0}^{m} \frac{(a q)_{n} t^{n} q^{n}}{(b q)_{n}} \\
=(1-b)-\frac{(a q)_{m+1} t^{m+1}}{(b q)_{m}} .
\end{gathered}
$$

Proof. In fact the left hand side of (4.1) is 


$$
\begin{aligned}
(1-t) & \sum_{n=0}^{m} \frac{(a q)_{n} t^{n}}{(b q)_{n}}-(b-a t q) \sum_{n=0}^{m} \frac{(a q)_{n} t^{n} q^{n}}{(b q)_{n}} \\
= & (1-t)-(b-a t q)+\sum_{n=1}^{m} \frac{(a q)_{n} t^{n}}{(b q)_{n}}-b \sum_{n=1}^{m} \frac{(a q)_{n} t^{n} q^{n}}{(b q)_{n}} \\
& -t\left[\sum_{n=1}^{m} \frac{(a q)_{n} t^{n}}{(b q)_{n}}-a q \sum_{n=1}^{m} \frac{(a q)_{n} t^{n} q^{n}}{(b q)_{n}}\right] \\
= & (1-b)-t(1-a q)+\sum_{n=1}^{m} \frac{(a q)_{n} t^{n}}{(b q)_{n-1}}-t \sum_{n=1}^{m} \frac{(a q)_{n+1} t^{n}}{(b q)_{n}} \\
= & (1-b)-\frac{(a q)_{m+1} t^{m+1}}{(b q)_{m}} .
\end{aligned}
$$

This proves (4.1).

In fact (4.1) can be obtained by simple manupulations in different ways.

Note. On taking limit as $m \rightarrow \infty$ in (4.1), we get the following identity given by N. J. Fine $[4$, p. 2]

$$
(1-t) \sum_{n=0}^{\infty} \frac{(a q)_{n} t^{n}}{(b q)_{n}}-(b-a t q) \sum_{n=0}^{\infty} \frac{(a q)_{n} t^{n} q^{n}}{(b q)_{n}}=(1-b) .
$$

5. An infinite continued fraction representation for the partial series of the type $\sum_{n=0}^{m}\left((\alpha)_{n} z^{n} /(\beta)_{n}\right)$

THEOREM I.

$$
A_{0}^{-1}(1-t) \sum_{n=0}^{m} \frac{(a q)_{n} t^{n} q^{n}}{(b q)_{n}}=\frac{1}{-\frac{(b-a t q)}{(1-t)}+P_{0}-\frac{Q_{0}}{P_{1}}-\frac{Q_{1}}{P_{2}}-\frac{Q_{2}}{P_{3}}-\cdots}
$$

where

$$
\begin{aligned}
P_{i} & =\left[\left(b-a t q^{i+1}\right) A_{i+1}+\left(1-t q^{i+1}\right) A_{i}\right] /\left(1-t q^{i}\right) A_{i+1}, \\
Q_{i} & =\left(b-a t q^{i+2}\right) A_{i} /\left(1-t q^{i}\right) A_{i+1}, \\
A_{i} & =\left[(1-b)-\frac{(a q)_{m+1} t^{m+1} q^{i(m+1)}}{(b q)_{m}}\right]
\end{aligned}
$$

and $i=0,1,2, \ldots$. 
The convergence of (5.1) can be dealt by Worpitzky's Theorem [8, p. 42].

Proof. Replacing $t$ as $t q$ in (4.1), we get

$$
\begin{gathered}
(1-t q) \sum_{n=0}^{m} \frac{(a q)_{n} t^{n} q^{n}}{(b q)_{n}}-\left(b-a t q^{2}\right) \sum_{n=0}^{m} \frac{(a q)_{n} t^{n} q^{2 n}}{(b q)_{n}} \\
=(1-b)-\frac{(a q)_{m+1} t^{m+1} q^{m+1}}{(b q)_{m}} .
\end{gathered}
$$

Solving (4.1) and (5.2), we get

$$
\begin{aligned}
{[(1-b)} & \left.-\frac{(a q)_{m+1} t^{m+1} q^{m+1}}{(b q)_{m}}\right](1-t) \sum_{n=0}^{m} \frac{(a q)_{n} t^{n}}{(b q)_{n}} \\
= & (b-a t q)\left\{(1-b)-\frac{(a q)_{m+1} t^{m+1} q^{m+1}}{(b q)_{m}}\right\} \\
& \left.+(1-t q)\left\{(1-b)-\frac{(a q)_{m+1} t^{m+1}}{(b q)_{m}}\right\}\right] \sum_{n=0}^{m} \frac{(a q)_{n} t^{n} q^{n}}{(b q)_{n}} \\
& -\left(b-a t q^{2}\right)\left[(1-b)-\frac{(a q)_{m+1} t^{m+1}}{(b q)_{m}}\right] \sum_{n=0}^{m} \frac{(a q)_{n} t^{n} q^{2 n}}{(b q)_{n}}
\end{aligned}
$$

Repeated iteration of (5.3), yields the following continued fraction

$$
\sum_{n=0}^{m} \frac{(a q)_{n} t^{n}}{(b q)_{n}} / \sum_{n=0}^{m} \frac{(a q)_{n} t^{n} q^{n}}{(b q)_{n}}=P_{0}-\frac{Q_{0}}{P_{1}}-\frac{Q_{1}}{P_{2}}-\frac{Q_{2}}{P_{3}}-\cdots
$$

From (4.1) and (5.4), we get the required continued fraction (5.1).

COROLlary. Taking the limit as $m \rightarrow \infty$ in (5.1), we get

$$
(1-b)^{-1}(1-t) \sum_{n=0}^{\infty} \frac{(a q)_{n} t^{n} q^{n}}{(b q)_{n}}=\frac{1}{-\frac{(b-a t q)}{(1-t)}+R_{0}-\frac{S_{0}}{R_{1}}-\frac{S_{1}}{R_{2}}-\frac{S_{2}}{R_{3}}-\cdots}
$$

where

$$
\begin{aligned}
& R_{i}=\left(1+b-t q^{i+1}-a t q^{i+1}\right)\left(1-t q^{i}\right)^{-1}, \\
& S_{i}=\left(b-a t q^{i+2}\right)\left(1-t q^{i}\right)^{-1}
\end{aligned}
$$

and $i=0,1,2, \ldots$ 
6. A finite continued fraction representation for the partial series of the type $\sum_{n=0}^{m}\left((\alpha)_{n} z^{n} /(\beta)_{n}\right)$

THEOREM II. For $m$ a finite positive integer, we have

$$
\begin{aligned}
& A_{0, m+2}^{-1}(1-t) \sum_{n=0}^{m} \frac{(a q)_{n} t^{n} q^{n}}{(b q)_{n}} \frac{1}{-\frac{(b-a t q)}{(1-t)}+P_{0, m+1}-\frac{Q_{0, m+1}}{P_{1, m}}-\frac{Q_{1, m}}{P_{2, m-1}}-\frac{Q_{2, m-1}}{P_{3, m-2}}-\cdots} \\
& \cdots-\frac{Q_{m-2,3}}{P_{m-1,2}}-\frac{Q_{m-1,2}}{1+\frac{(1-a q) t q^{m}}{(1-b q)}} \\
& \frac{1}{1}
\end{aligned}
$$

where

$$
\begin{aligned}
& P_{i, j}=\left[\left(b-a t q^{i+1}\right) A_{i+1, j}+\left(1-t q^{i+1}\right) A_{i, j+1}\right] /\left(1-t q^{i}\right) A_{i+1, j}, \\
& Q_{i, j}=\left(b-a t q^{i+2}\right) A_{i, j+1} /\left(1-t q^{i}\right) A_{i+1, j}, \\
& A_{i, j}=\left[(1-b)-\frac{(a q)_{j} t^{j} q^{i j}}{(b q)_{j-1}}-\frac{\left(b-a t q^{i+1}\right)(a q)_{j-1} t^{j-1} q^{j(i+1)-i-1}}{(b q)_{j-1}}\right]
\end{aligned}
$$

and $i=0,1,2, \ldots,(m-1), j=2,3,4, \ldots,(m+1)$.

Proof. From (4.1), we get

$$
\begin{aligned}
(1-t) & \sum_{n=0}^{m+1} \frac{(a q)_{n} t^{n}}{(b q)_{n}}-(b-a t q) \sum_{n=0}^{m} \frac{(a q)_{n} t^{n} q^{n}}{(b q)_{n}} \\
= & (1-b)-\frac{(a q)_{m+2} t^{m+2}}{(b q)_{m+1}}-\frac{(b-a t q)(a q)_{m+1} t^{m+1} q^{m+1}}{(b q)_{m+1}} .
\end{aligned}
$$

Replace $t$ as $t q$ and $m$ as $(m-1)$ in (6.2), we get

$$
\begin{aligned}
(1-t q) & \sum_{n=0}^{m} \frac{(a q)_{n} t^{n} q^{n}}{(b q)_{n}}-\left(b-a t q^{2}\right) \sum_{n=0}^{m-1} \frac{(a q)_{n} t^{n} q^{2 n}}{(b q)_{n}} \\
= & (1-b)-\frac{(a q)_{m+1} t^{m+1} q^{m+1}}{(b q)_{m}}-\frac{\left(b-a t q^{2}\right)(a q)_{m} t^{m} q^{2 m}}{(b q)_{m}} .
\end{aligned}
$$

Solving (6.2) and (6.3), we get 


$$
\begin{aligned}
{[(1-b)} & \left.-\frac{(a q)_{m+1} t^{m+1} q^{m+1}}{(b q)_{m}}-\frac{\left(b-a t q^{2}\right)(a q)_{m} t^{m} q^{2 m}}{(b q)_{m}}\right](1-t) \sum_{n=0}^{m+1} \frac{(a q)_{n} t^{n}}{(b q)_{n}} \\
= & (b-a t q)\left\{(1-b)-\frac{(a q)_{m+1} t^{m+1} q^{m+1}}{(b q)_{m}}-\frac{\left(b-a t q^{2}\right)(a q)_{m} t^{m} q^{2 m}}{(b q)_{m}}\right\} \\
& \left.+(1-t q)\left\{(1-b)-\frac{(a q)_{m+2} t^{m+2}}{(b q)_{m+1}}-\frac{(b-a t q)(a q)_{m+1} t^{m+1} q^{m+1}}{(b q)_{m+1}}\right\}\right] \\
& \times \sum_{n=0}^{m} \frac{(a q)_{n} t^{n} q^{n}}{(b q)_{n}} \\
& -\left(b-a t q^{2}\right)\left[(1-b)-\frac{(a q)_{m+2} t^{m+2}}{(b q)_{m+1}}-\frac{(b-a t q)(a q)_{m+1} t^{m+1} q^{m+1}}{(b q)_{m+1}}\right] \\
& \times \sum_{n=0}^{m-1} \frac{(a q)_{n} t^{n} q^{2 n}}{(b q)_{n}} .
\end{aligned}
$$

Repeated iteration of (6.4) yields the following continued fraction

$$
\begin{aligned}
& \sum_{n=0}^{m+1} \frac{(a q)_{n} t^{n}}{(b q)_{n}} / \sum_{n=0}^{m} \frac{(a q)_{n} t^{n} q^{n}}{(b q)_{n}} \\
& \quad=P_{0, m+1}-\frac{Q_{0, m+1}}{P_{1, m}}-\frac{Q_{1, m}}{P_{2, m-1}}-\frac{Q_{2, m-1}}{P_{3, m-2}}-\cdots-\frac{Q_{m-2,3}}{P_{m-1,2}}-\frac{Q_{m-1,2}}{1+\frac{(1-a q) t q^{m}}{(1-b q)}}
\end{aligned}
$$

From (6.2) and (6.5) we get the required continued fraction (6.1).

\section{Certain special cases of our results given in the previous two sections}

(i) Putting $a=0, b=-1$ and $t=-q$ in (5.1), we get

$$
B_{0}^{-1}(1+q)\left[(1+q) f_{m}(q)-2 q\right]=\frac{1}{\frac{1}{(1+q)}+E_{0}-\frac{F_{0}}{E_{1}}-\frac{F_{1}}{E_{2}}-\frac{F_{2}}{E_{3}}-\cdots}
$$

where

$$
\begin{aligned}
E_{i} & =\left[\left(1+q^{i+2}\right) B_{i}-B_{i+1}\right] /\left(1+q^{i+1}\right) B_{i+1}, \\
F_{i} & =-B_{i} /\left(1+q^{i+1}\right) B_{i+1}, \\
B_{i} & =\left[2+\frac{(-1)^{m} q^{(i+1)(m+1)}}{(-q)_{m}}\right]
\end{aligned}
$$

and $i=0,1,2, \ldots$ 
(ii) Taking limit $m \rightarrow \infty$ in (7.1), we get

$$
\frac{(1+q)}{2}[(1+q) f(q)-2 q]=\frac{1}{\frac{1}{(1+q)}+G_{0}-\frac{H_{0}}{G_{1}}-\frac{H_{1}}{G_{2}}-\frac{H_{2}}{G_{3}}-\cdots}
$$

where

$$
\begin{aligned}
& G_{i}=q^{i+2}\left(1+q^{i+1}\right)^{-1}, \\
& H_{i}=-\left(1+q^{i+1}\right)^{-1},
\end{aligned}
$$

and $i=0,1,2, \ldots$

(iii) Putting $a=0, b=-1, t=-q$ in (6.1), we get

For $m$ a finite positive integer,

$$
\begin{aligned}
& \text { 7.3) } B_{0, m+2}^{-1}(1+q)\left[(1+q) f_{m}(q)-2 q\right] \\
& \frac{1}{(1+q)}+E_{0, m+1}-\frac{F_{0, m+1}}{E_{1, m}}-\frac{F_{1, m}}{E_{2, m-1}}-\frac{F_{2, m-1}}{E_{3, m-2}}-\cdots-\frac{F_{m-2,3}}{E_{m-1,2}}-\frac{F_{m-1,2}}{1-\frac{q^{m+1}}{(1+q)}} \\
& \frac{1}{1}
\end{aligned}
$$

where

$$
\begin{aligned}
& E_{i, j}=\left[\left(1+q^{i+2}\right) B_{i, j+1}-B_{i+1, j}\right] /\left(1+q^{i+1}\right) B_{i+1, j}, \\
& F_{i, j}=-B_{i, j+1} /\left(1+q^{i+1}\right) B_{i+1, j}, \\
& B_{i, j}=\left[2-\frac{(-1)^{j} q^{i j}}{(-q)_{j-1}}-\frac{(-1)^{j} q^{(i+2)(j-1)}}{(-q)_{j-1}}\right]
\end{aligned}
$$

and $i=0,1,2, \ldots,(m-1), j=2,3,4, \ldots,(m+1)$.

(iv) Replacing $a$ by $a / q$, then putting $t=z / a, b=0$ and letting limit $a \rightarrow \infty$ in (6.1) and (6.3) we get the continued fraction representation of the partial series $\sum_{n=0}^{m}(-1)^{n} z^{n} q^{n(n+1) / 2}$ of the following Eisenstein continued fraction representation $[2$, p. 66]:

$$
\sum_{n=0}^{\infty}(-1)^{n} z^{n} q^{n(n+1) / 2}=\frac{1}{1}+\frac{a_{1}}{1}+\frac{a_{2}}{1}+\cdots
$$

where 


$$
\begin{aligned}
& a_{2 k}=-z q^{k}\left(1-q^{k}\right), \quad k \geq 1 \\
& a_{2 k+1}=q^{2 k+1} z, \quad k \geq 0 .
\end{aligned}
$$

(v) Putting $b=q$ in (5.1) and $b=1$ in (6.1), we get the infinite and finite continued fraction representations respectively for the partial series of the type $\sum_{n=0}^{m}\left((\alpha)_{n} z^{n} /(q)_{n}\right)$.

Similarly, one can get infinite and finite continued fraction representations for the other partial mock theta functions $\phi_{m}(q), \psi_{m}(q), \chi_{m}(q), \omega_{m}(q), v_{m}(q)$, $\rho_{m}(q), \phi_{L m}(q), \sigma_{L m}(q), \mu_{L m}(q), \gamma_{L m}(q)$, and Jacobi's partial theta functions $\theta_{2, m}(0), \theta_{3, m}(0), \theta_{4, m}(0)$.

\section{Concluding remarks}

It may be observed that these continued fractions owe their importance in finding the bounds for the partial and the infinite forms of the various third order mock theta functions. In the pious hope that one may be able to prove through the continued fraction representations the second condition [1] of validity for being a mock theta function, namely. "There is no single theta function which works for all $\xi$, i.e. for every $\theta$-function $\theta(q)$ there is some root of unity $\xi$ for which $f(q)$ minus the theta function $\theta(q)$ is unbounded as $q \rightarrow \xi$ radially."

It may be remarked that the above condition has neither be proved or disproved, thus far. The above study has been continued in a further communication for the fifth and seventh order mock theta functions also.

Acknowledgement. The author is grateful to Prof. R. P. Agarwal for his kind guidance during the preparation of this paper. This work has been completed under the Research Project No. CST/D-3197 financially supported by the U.P. Council of Science and Technology, to whom I am grateful.

\section{REFERENCES}

[1] R. P. Agarwal, Mock theta functions - an analytical point of view, Proc. Nat. Acad. Sci. India Sect. A, 64 (1994), 95-107.

[2] R. P. Agarwal, Resonance of Ramanujan's Mathematics Vol. III, New Age International Publishers, New Delhi, 1999.

[ 3 ] G. E. Andrews, Ramanujan's "lost" notebook VII. The sixth order mock theta and functions, Adv. Math., 89 (1991), 60-105.

[4] N. J. FINE, Basic hypergeometric series and applications, Math. Surveys Monogr. 27, American Mathematical Society, Providence, 1988.

[ 5 ] A. Gupta, On certain Ramanujan's mock theta functions, Proc. Indian Acad. Sci. Math. Sci., 103 (1993), 257-267.

[6] E. D. Rainville, Special Functions, Chelsea Publishing, Bronx, 1960.

[7] S. Ramanujan, The Lost Notebook and Other Unpublished Papers, Narosa Publishing House, New Delhi, 1988. 
[ 8 ] H. S. WALl, Analytic Theory of Continued Fractions, D. Van Nostrand Company, New York, 1948.

Department of Mathematics \& Astronomy LUCKNOW UNIVERSITY

LUCKNOW (U.P.)

INDIA 